\title{
Apego aos Pais, Relações Românticas, Estilo de Vida, Saúde Física e Mental em Universitários
}

\author{
Attachment to Parents, Romantic Relationships, Lifestyle, \\ Physical and Mental Health in University Students \\ Maria da Graça Pereira*, Gabriela Ferreira \& Ana Cristina Paredes \\ Universidade do Minho, Braga, Distrito de Braga, Portugal
}

\begin{abstract}
Resumo
Pretendeu-se conhecer a relação entre apego aos pais, características das relações românticas, sintomatologia física, estilo de vida e morbilidade psicológica em 250 estudantes universitários, entre os 17 e 29 anos, que responderam a instrumentos de auto relato. Concluiu-se que o apego aos pais nos jovens adultos se relaciona com a sintomatologia física, a morbilidade psicológica e características das relações amorosas. O envolvimento numa relação romântica reflecte-se na saúde, estando associado ao estilo de vida saudável e menos sintomas físicos. Exploraram-se os preditores da sintomatologia física e estilo de vida, e analisou-se a influência do sexo, tipo de família e nível socioeconómico nas variáveis em estudo. Assim, podem retirar-se implicações para o desenvolvimento de intervenções com jovens e pais.

Palavras-chave: Apego, relações românticas, estilo de vida, sintomatologia física, morbilidade psicológica.
\end{abstract}

\begin{abstract}
This study focused on the understanding of the relationship among attachment to parents, characteristics of romantic relationships, physical symptoms, lifestyle and psychological morbidity in 250 university students, aged between 17 and 29 years $(M=20.88 ; S D=2.03)$, who answered self-report questionnaires. We concluded that attachment to parents for young adults is related to physical symptoms, psychological morbidity and characteristics of romantic relationships. Being in a romantic relationship has effects on health, since it is related to a healthier lifestyle and less physical symptoms. We explored the predictors of physical symptoms and lifestyle, and analyzed the influence of sex, type of family and socioeconomic status on the variables studied. As a result, implications can be drawn to develop interventions with youngsters and parents.

Keywords: Attachment, romantic relationships, lifestyle, physical symptoms, psychological morbidity.
\end{abstract}

Na sua interação com os cuidadores, as crianças desenvolvem modelos internos dinâmicos - representações mentais acerca de si, dos outros e do que podem esperar desta relação. Se a criança perceciona a relação com o cuidador como adequada às suas necessidades, forma uma imagem de si como merecedora de amor e atenção, e do cuidador como responsivo e confiável; por outro lado, se as suas necessidades não são satisfeitas, cria uma imagem de si como pouco eficaz e do outro como indisponível (Ainsworth, Blehar, Waters, \& Wall, 1978; Berlin, Cassidy, \& Appleyard, 2008; Bowlby, 1998; Holmes, 1993). É através dos modelos internos dinâmicos que os esquemas precoces de apego se refletem na personalidade dos indivíduos e, consequentemente, nas suas relações futuras (N.

\footnotetext{
" Endereço para correspondência: Universidade do Minho, Escola de Psicologia, Campus de Gualtar, Braga, Distrito de Braga, Portugal 4710-057. E-mail: gracep@psi.uminho.pt, gabriela.m.m.ferreira@gmail. com e anacristinaparedes@gmail.com
}

L. Collins \& Read, 1990; Hazan \& Shaver, 1987; Morgan \& Shaver, 1999), permanecendo ativos não só na infância, mas também na idade adulta (Bowlby, 1984, 1985). Assim, nesta fase podem-se encontrar os mesmos três padrões de apego identificados por Ainsworth et al. (1978) existentes na infância: seguro, ansioso-ambivalente e evitante que influenciam as estratégias de regulação emocional face a outros significativos e, assim, contribuem para o modo como os indivíduos se envolverão em relações amorosas, devido à sua função organizadora do comportamento $(\mathrm{N}$. L. Collins \& Read, 1990; Hazan \& Shaver, 1987; Myers \& Vetere, 2002; Morgan \& Shaver, 1999).

Deste modo, indivíduos com diferentes padrões de apego envolvem-se de forma diferente nas relações românticas (W. A. Collins \& Sroufe, 1999; Morgan \& Shaver, 1999). Os indivíduos com apego seguro tendem a ter uma visão mais romântica das relações, enquanto que os mais ansiosos e inseguros tendem a ser mais obsessivos e dependentes, estabelecendo relacionamentos com baixos níveis de confiança, satisfação e compromisso (Altin \& Terzi, 2010; N. L. Collins \& Read, 1990; Simpson, 1990). 
Vários estudos têm vindo a demonstrar que o apego e as relações românticas têm influência ao nível da saúde dos indivíduos, nomeadamente no que se refere ao seu estilo de vida, à perturbação emocional e aos sintomas físicos que relatam. A literatura tem mostrado que as relações de intimidade possuem benefícios ao nível da promoção de comportamentos protectores da saúde (i.e. estilo de vida), definido como um conjunto de atitudes e comportamentos que orientam o indivíduo a determinados hábitos de vida (Green \& Anderson, 1986). Assim, indivíduos envolvidos em relações amorosas com maiores níveis de intimidade têm mais probabilidade de aderir positivamente a apelos para comportamentos de saúde e adotar menos comportamentos de risco (Braithwaite, Delevi, \& Fincham, 2010; Dennis, 2006). Também Ribeiro e Sousa (2002) salientam o efeito benéfico de um estilo de apego seguro na adoção de comportamentos de saúde e, consequentemente, no bem-estar dos indivíduos, verificando-se diferenças de género a este nível, com as raparigas a evidenciarem comportamentos mais saudáveis; e, por outro lado, uma relação consistente entre um estilo de apego inseguro e a morbilidade psicológica.

Segundo Bowlby (1985), a perturbação emocional resulta de um percurso anormal no desenvolvimento psicológico que se deve à formação de representações negativas acerca das relações com os outros que influenciam avaliações irrealistas, o que ilustra a importância das relações precoces ao nível da morbilidade psicológica (Atkinson, 1997). Tem sido sugerido que indivíduos com apego inseguro apresentam maiores níveis de sintomatologia depressiva (Altin \& Terzi, 2010; Simpson, 1990). Pelo contrário, a sintomatologia depressiva parece ser menor nos indivíduos envolvidos numa relação amorosa, o que se deve em parte ao apoio do parceiro, sendo os homens mais suscetíveis a esta influência (Braithwaite et al., 2010; Simon \& Barrett, 2010). Assim, a literatura indica claramente que o envolvimento numa relação amorosa traz benefícios emocionais aos envolvidos (Kiecolt-Glaser \& Newton, 2001). Em Portugal, são escassos os estudos que analisam a relação entre o apego, a saúde física e a saúde psicológica (Monteiro, Tavares, \& Pereira, 2007). Um estudo realizado com estudantes universitários portugueses mostrou que um estilo de apego evitante se relacionava com mais sintomatologia psicopatológica e menor bem-estar (Monteiro et al., 2007).

Também a sintomatologia física tem vindo a ser relacionada com a qualidade do envolvimento amoroso, sendo que os indivíduos mais satisfeitos com as suas relações relatam menos sintomas físicos (Markey, Markey, \& Gray, 2007). Hale (2006) identificou a intimidade e o conflito na relação como preditores da saúde física e Kiecolt-Glaser e Newton (2001) mostraram que o envolvimento numa relação amorosa beneficia a saúde física dos envolvidos.

Apesar do reduzido número de estudos que exploram a relação entre saúde e estilos de apego (Myers \& Vetere, 2002), alguns autores apontam para a existência de uma correspondência entre estas variáveis, sugerindo que a sintomatologia física se relaciona positivamente com os estilos ansiosos e evitantes e negativamente com o estilo seguro (Wearden, Lamberton, Crook, \& Walsh, 2005). Pelo contrário, Myers e Vetere (2002) concluíram que o estilo de apego no adulto não está relacionado com sintomas físicos. A grande variedade de resultados obtidos pode, em parte, ser explicada pela diversidade de instrumentos e perspectivas teóricas que enquadram a classificação do apego. Apesar de a relação entre saúde física e apego não ser muito clara, esta controvérsia dissipa-se quando falamos de perturbação emocional favorecida pelos padrões de apego inseguro (Ribeiro \& Sousa, 2002).

Este estudo teve por objetivo (a) compreender a relação entre o apego aos pais, as características da relação romântica, a sintomatologia física, a morbilidade psicológica e o estilo de vida; (b) averiguar a existência de diferenças entre os jovens que estão ou não envolvidos numa relação amorosa, ao nível das variáveis em estudo; (c) explorar os preditores da sintomatologia física e do estilo de vida. Foram ainda realizadas análises exploratórias para averiguar a influência do sexo, tipo de família e nível socioeconómico (NSE) nas variáveis em estudo. Este estudo configura-se inovador na medida em que pretende alargar o corpo de investigação sobre a relação entre apego, relações românticas e saúde, numa faixa etária pouco estudada neste âmbito.

\section{Método}

\section{Participantes}

A amostra é constituída por 250 jovens universitários duma Universidade Central pública do Norte de Portugal, na sua maioria de nacionalidade portuguesa $(98,4 \%)$, sendo $66 \%$ do sexo feminino. A sua idade varia entre 17 e 29 anos $(M=20,88, D P=2,03)$. A maioria dos participantes tem irmãos $(86,4 \%)$. No que se refere ao tipo de família, $85,9 \%$ pertencem a uma família intacta; $51,6 \%$ dos indivíduos estão envolvidos numa relação de compromisso e a média da duração dessas relações é de 27,62 meses $(D P=20,18) ; 96,3 \%$ dessas relações foram heterossexuais. Nesta amostra $28 \%$ dos participantes frequentam cursos de Ciências Económicas e Políticas, 17,6\% cursos de Engenharia, 15,6\% de Ciências Aplicadas, 10,8\% de Ensino, $8,4 \%$ de Ciências Sociais, $8 \%$ de Ciências Humanas, $4 \%$ de Ciências da Saúde e 1,6\% de Artes.

\section{Instrumentos}

Questionário Sociodemográfico (Pereira, Ferreira, \& Paredes, 2010). Este questionário tem por objetivo recolher informação social e demográfica de modo a caracterizar a amostra (e.g., sexo, idade, tipo de família, envolvimento numa relação amorosa atualmente e nível socioeconómico). O nível socioeconómico foi avaliado através da Graffar que indica a classe social da família de acordo com cinco critérios: profissão, nível de instrução, fontes de rendimento familiar, conforto do alojamento e aspeto do bairro onde habita. Um resultado baixo é indicativo de maior nível socioeconómico. 
Relationship Rating Form (RRF; Davis, 1996; versão portuguesa de Lind, 2008). Este questionário avalia as características e a qualidade das relações amorosas e é constituído por 68 itens que se subdividem em seis escalas - Viabilidade, Intimidade, Paixão, Apoio, Satisfação Global e Conflito/Ambivalência - e 20 facetas. Há ainda quatro subescalas que não se inserem nas seis escalas principais: Manutenção, Compromisso, Coerção e Igualdade. Um resultado elevado é indicativo de maior satisfação com a relação (Moreira et al., 2006). No presente estudo, os alfas obtidos foram de 0,91 para os homens e 0,92 para as mulheres ao nível da escala total. Relativamente às escalas e dimensões, o alfa das escalas utilizadas variou entre 0,94 e 0,70 , com exceção da subescala Manutenção que obteve um alfa de 0,51 e que por esse motivo não foi integrada no teste de hipóteses (Field, 2009).

Questionário de Vinculação ao Pai e á Mãe (QVPM). O QVPM (versão revista de Matos \& Costa, 2001) avalia a qualidade do apego dos jovens ao pai e à mãe. A versão utilizada é composta por 30 itens, divididos em três subescalas: Inibição da Exploração e Individualidade (IEI), Qualidade do Laço Emocional (QLE) e Ansiedade de Separação e Dependência (ASD). Cada subescala é composta por 10 itens. A IEI avalia a perceção que o indivíduo tem de restrições à expressão da sua individualidade própria, seja pelas dificuldades sentidas na emissão de pontos de vista ou opiniões divergentes das da figura parental, seja pela ausência de apoio a iniciativas de exploração ou pela interferência, não desejada, em questões que considera pessoais. (Matos, 2002)

A QLE avalia o quão importante são as figuras parentais como figuras de apego ao nível do desenvolvimento do indivíduo e a ASD refere-se à avaliação dos indivíduos acerca das situações de separação da figura de apego (Matos, 2002; Rocha, 2008). Resultados elevados são indicativos de maior apego na dimensão considerada.

Os valores de consistência interna (alfa de Cronbach) do QVPM numa amostra de jovens com idades entre os 13 e os 23 anos variaram entre 0,79 e 0,89 para a mãe e entre 0,78 e 0,94 para o pai (Rocha, 2008). No presente estudo, os valores de alfa de Cronbach variam entre 0,81 e 0,87 para o apego em relação á mãe e entre 0,84 e 0,92 para o apego em relação ao pai.

Questionário de Estilo de Vida $(\mathrm{QEV}$; versão de investigação de Pereira \& Pedras, 2008). Este instrumento permite avaliar comportamentos e hábitos de saúde associados a um estilo de vida saudável. Um resultado elevado corresponde a um estilo de vida mais saudável (Pedras, 2009). Na presente amostra, o valor de alfa de Cronbach foi de 0,69 .

Rotterdam Symptom Checklist (RSCL; De Haes, Van Knippenberg, \& Neijt, 1990; versão portuguesa de Santos et al., 1994). A sintomatologia física foi avaliada através da escala física da RSCL que inclui 23 itens que avaliam sintomatologia física comum (e.g.: dores, náuseas, fadiga, dores de barriga...). Um resultado mais elevado é indicativo de maior sintomatologia física (De Haes et al., 1996).
Na presente amostra o alfa obtido foi de 0,87 .

Hospital Anxiety and Depression Scale (HADS; Zigmond \& Snaith, 1983; versão portuguesa de McIntyre, Pereira, Soares, Gouveia, \& Silva, 1999). Esta escala avalia a ansiedade e a depressão em populações clinicas e não clinicas. É constituída por 14 itens, agrupados em duas subescalas: ansiedade e depressão. Um resultado elevado é indicativo de maior ansiedade e depressão. Pode igualmente ser calculada uma pontuação total da escala como indicador de morbilidade psicológica (Gouveia, 2004).

Dado este instrumento não ter sido validado numa amostra não clínica, realizou-se uma análise fatorial em componentes principais que permitiu extrair dois fatores, tal como proposto pelos autores originais, explicando $40,66 \%$ da variância total. Ao contrário do que se verificou na versão original, os itens 6,8 e 10 saturaram no fator 1 (ansiedade) em vez do fator 2 (depressão). Assim, o alfa de Cronbach obtido para a escala total foi de 0,80 , e de 0,82 para a subescala ansiedade e 0,50 para a subescala depressão. Optou-se por integrar nas análises apenas a escala total, indicadora de morbilidade psicológica.

\section{Procedimento}

Neste estudo foi utilizada uma amostra voluntária de jovens duma universidade pública do Norte de Portugal. Foi obtida aprovação da universidade e os estudantes foram informados acerca dos objetivos do estudo e do caráter voluntário da sua participação, tendo assinado o consentimento informado e preenchido individualmente a bateria de avaliação.

\section{Análise de Dados}

Foram realizadas análises de variância multivariada (MANOVA) para averiguar as diferenças em função do sexo, tipo de família, nível socioeconómico (NSE) e do envolvimento ou não numa relação amorosa ao nível das variáveis em estudo, quando os pressupostos de normalidade da distribuição e homogeneidade da variância se encontravam presentes (Field, 2009; Maroco, 2007). Quando isso não acontecia recorreu-se aos testes não paramétricos equivalentes (testes de Mann-Whitney). Foram testados vários modelos tendo em consideração a multicolinearidade entre as variáveis dependentes, nomeadamente o modelo de apego ao pai e outro de apego à mãe, e ainda modelo com as variáveis da relação romântica. O NSE e o tipo de família foram categorizados da seguinte forma NSE elevado versus NSE médio-baixo e família intacta versus monoparental, respetivamente. Para relacionar as variáveis em estudo utilizou-se o Coeficiente de Correlação de Pearson.

Efetuaram-se também regressões hierárquicas (método Enter), para prever a sintomatologia física e o estilo de vida. As variáveis preditoras foram introduzidas em blocos distintos. Assim, no primeiro bloco foram introduzidas as variáveis sociodemográficas: sexo, e tipo de família (variáveis dummy) e a variável idade (intervalar); e no segundo, as variáveis de apego à mãe ou ao pai - a Ini- 
Pereira, M. G., Ferreira, G. \& Paredes, A. C. (2013). Apego aos Pais, Relações Românticas, Estilo de Vida, Saúde Física e Mental em Universitários.

bição de Exploração e Individualidade (IEI), a Qualidade do Laço Emocional (QLE) e a Ansiedade de Separação e Dependência (ASD); a morbilidade psicológica e o estilo de vida ou a sintomatologia física, consoante a variável dependente. No que se refere ao estilo de vida, foram testados dois modelos separadamente, um com as variáveis de apego à mãe e outro com as variáveis de apego ao pai dada a multicolinearidade entre estes dois conjuntos de variáveis (Field, 2009).

\section{Resultados}

Influência do Sexo, Tipo de Familia e Nivel Socioeconómico (NSE) nas Variáveis em Estudo

Verificaram-se diferenças significativas entre homens e mulheres ao nível da sintomatologia física $[F(1,243)=$ $14,16, p<0,001]$, morbilidade psicológica $[F(1,243)=$ $6,14, p=0,014]$, Ansiedade de Separação e Dependência (ASD) em relação ao pai $[F(1,234)=4,18, p=0,042] \mathrm{e}$ à mãe $[F(1,243)=8,44, p=0,004]$, e estilo de vida $[F$ $(1,243)=30,03, p<0,001]$. Assim, as mulheres apresentam mais sintomas físicos; maior ansiedade, morbilidade psicológica e ASD em relação ao pai e à mãe; e um estilo de vida mais saudável, do que os homens. Não se encontraram diferenças significativas nas restantes variáveis. Verificaram-se diferenças entre indivíduos de famílias intactas versus famílias monoparentais ao nível da inibição de exploração e individualidade (IEI) em relação à mãe $[F(1,241)=4,83, p=0,029]$. Jovens de famílias monoparentais percepcionam maior IEI por parte da mãe, do que os que pertencem a famílias intactas. Não se encontraram diferenças significativas nas restantes variáveis.

Também se verificaram diferenças ao nível da ASD em relação à mãe $[F(1,243)=7,16, p=0,008]$ e da perceção de conflito na relação romântica $[F(1,127)=5,52$, $p=0,020]$, em função do nível socioeconómico (NSE). Assim, jovens de família cujo NSE é médio/baixo per- cecionam maior ASD em relação à mãe e maior conflito nas relações românticas, do que os de famílias de NSE elevado. Não se encontraram diferenças significativas nas restantes variáveis.

Relação entre Apego aos Pais, Características da Relação Romântica, Sintomatologia Física e Morbilidade Psicológica e Estilo de Vida

Os resultados revelaram uma correlação positiva significativa da sintomatologia física com a IEI por parte do pai $(r=0,28, p<0,001)$ e da mãe $(r=0,23, p<0,001)$. Mais sintomatologia física encontra-se associada à perceção de maior IEI por parte do pai e mãe.

Verificou-se uma correlação negativa significativa entre a morbilidade psicológica $\mathrm{e} \mathrm{a}$ QLE em relação à mãe $(r$ $=-0,14, p=0,031)$ e pai $(r=-0,13, p=0,046)$; e positiva com a IEI por parte do pai $(r=0,33, p<0,001)$, mãe $(r=$ $0,29, p<0,001)$ e com o conflito na relação romântica $(r$ $=0,19, p=0,033)$. Assim, menor QLE em relação à mãe e pai e maior IEI, por parte do pai e mãe, estão associadas a maior morbilidade psicológica. Também maior conflito nas relações românticas está associado a maior morbilidade psicológica. Encontrou-se ainda uma correlação negativa significativa entre a satisfação global com a relação e a IEI por parte da mãe $(r=-0,19, p=0,030)$. Não se encontraram relações significativas entre as restantes variáveis.

Diferenças entre Estar ou Não Envolvido numa Relação Amorosa ao Nível das Variáveis em Estudo

Os resultados revelaram diferenças significativas entre jovens que estão envolvidos numa relação amorosa versus os que não estão ao nível do seu estilo de vida $[F(1,243)$ $=5,67, p=0,018]$ e sintomatologia física $[F(1,243)=$ $4,69, p=0,031]$. Assim, os jovens numa relação amorosa apresentam um estilo de vida mais saudável e menos sintomatologia física. Não se encontraram diferenças significativas nas restantes variáveis.

Tabela 1

Resultados do Teste MANOVA ao Nivel das Variáveis de Vinculação à Mãe, Estilo de Vida, Sintomatologia Física e Morbilidade Psicológica em Função do Envolvimento numa Relação Romântica

\begin{tabular}{lccccc}
\hline & $\begin{array}{c}\text { Não envolvido numa } \\
\text { relação romântica } \\
n=118\end{array}$ & $\begin{array}{c}\text { Envolvido numa } \\
\text { relação romântica } \\
n=127\end{array}$ & & & \\
& Média $(D P)$ & Média $(D P)$ & $F(1,243)$ & $\eta$ & $p$ \\
\hline IEI mãe & $28,45(10,13)$ & $27,03(9,90)$ & 1,23 & 0,005 & 0,27 \\
QLE mãe & $53,57(5,79)$ & $53,88(5,87)$ & 0,18 & 0,001 & 0,67 \\
ASD mãe & $37,68(8,86)$ & $38,42(8,34)$ & 0,45 & 0,002 & 0,50 \\
Estilo de vida & $69,48(8,16)$ & $71,81(7,19)$ & 5,67 & 0,023 & $\mathbf{0 , 0 2}$ \\
Sintomatologia física & $38,79(10,56)$ & $36,20(8,09)$ & 4,69 & 0,019 & $\mathbf{0 , 0 3}$ \\
Morbilidade psicológica & $11,73(5,88)$ & $10,35(5,74)$ & 3,47 & 0,014 & 0,06 \\
\hline
\end{tabular}


Variáveis Preditoras da Sintomatologia Física e do Estilo de Vida

Verificou-se que o sexo, o tipo de família, o estilo de vida e a morbilidade psicológica predizem significativamente a sintomatologia física explicando $40 \%$ da variância. O sexo masculino $(\beta=-0,19, t=-3,47, p=0,001)$ e um estilo de vida saudável $(\beta=-0,12, t=-2,10, p=0,04)$ predizem menos sintomas físicos, enquanto que pertencer a uma família monoparental $(\beta=0,10, t=2,03, p=0,04)$ e apresentar mais morbilidade psicológica $(\beta=0,56, t=$ $10,51, p<0,001)$ predizem mais sintomatologia física.

No modelo que integra as variáveis de apego à mãe, constatou-se que o sexo, a idade, a IEI, por parte da mãe, e a sintomatologia física são preditores significativos do estilo de vida explicando $17,6 \%$ da variância. Assim, ser do sexo masculino $(\beta=-0,33, t=-5,31, p<0,001)$, ter mais idade $(\beta=-0,14, t=-2,28, p=0,02)$, perceção de maior IEI por parte da mãe $(\beta=-0,15, t=-2,16, p=0,03)$ e mais sintomatologia física $(\beta=-0,16, t=-2,09, p=0,04)$ predizem um estilo de vida menos saudável. No modelo que inclui as variáveis de apego ao pai, verificou-se que o sexo masculino $(\beta=-0,33, t=-5,19, p<0,001)$, mais idade $(\beta=-0,15, t=-2,36, p=0,02)$, perceção de maior IEI por parte do pai $(\beta=-0,15, t=-2,11, p=0,04)$, predizem significativamente um estilo de vida menos saudável explicando $15,8 \%$ da variância.

Tabela 2

Regressões Hierárquicas: Preditores da Sintomatologia Física ( $N=243)$

\begin{tabular}{lcccc}
\hline Preditor & $\Delta \mathrm{R}^{2}$ & $B$ & $E P B$ & $B$ \\
\hline Bloco 1 & 0,07 & & & \\
$\quad$ Sexo & & $-4,57$ & 1,27 & $-0,23^{* * *}$ \\
$\quad$ Idade & & $-0,10$ & 0,30 & $-0,02$ \\
$\quad$ Tipo de família & 2,77 & 1,72 & 0,10 \\
Bloco 2 & 0,36 & & \\
Sexo & & $-3,80$ & 1,10 & $-0,19^{* *}$ \\
Idade & & $-0,22$ & 0,24 & $-0,05$ \\
Tipo de família & 2,82 & 1,39 & $0,10^{*}$ \\
IEI mãe & & 0,05 & 0,06 & 0,06 \\
QLE mãe & & 0,08 & 0,10 & 0,05 \\
ASD mãe & & $-0,05$ & 0,06 & $-0,04$ \\
Estilo de vida & $-0,14$ & 0,07 & $-0,12^{*}$ \\
MorbilidadePsicológica & & 0,91 & 0,09 & $0,56^{* * *}$ \\
\hline
\end{tabular}

Notas. IEI- Inibição da Exploração e Individualidade; QLE- Qualidade do Laço Emocional; ASD- Ansiedade de Separação e Dependência.

$* p<0,05, * * p<0,01, * * * p<0,001$. 
Pereira, M. G., Ferreira, G. \& Paredes, A. C. (2013). Apego aos Pais, Relações Românticas, Estilo de Vida, Saúde Física e Mental em Universitários.

Tabela 3

Regressões Hierárquicas: Preditores do Estilo de Vida Incluindo as Variáveis de Apego à Mãe (N=243)

\begin{tabular}{|c|c|c|c|c|}
\hline Preditor & $\Delta \mathrm{R}^{2}$ & $B$ & $E P B$ & $B$ \\
\hline Bloco 1 & 0,13 & & & \\
\hline Sexo & & $-5,12$ & 1,01 & $-0,31 * * *$ \\
\hline Idade & & $-0,54$ & 0,24 & $-0,14 *$ \\
\hline Tipo de família & & 0,58 & 1,37 & 0,03 \\
\hline Bloco 2 & 0,07 & & & \\
\hline Sexo & & $-5,45$ & 1,03 & $-0,33 * * *$ \\
\hline Idade & & $-0,53$ & 0,23 & $-0,14^{*}$ \\
\hline Tipo de família & & 1,46 & 1,35 & 0,07 \\
\hline IEI mãe & & $-0,12$ & 0,05 & $-0,15^{*}$ \\
\hline QLE mãe & & 0,03 & 0,10 & 0,02 \\
\hline ASD mãe & & 0,04 & 0,06 & 0,04 \\
\hline Sintomatologia Física & & $-0,13$ & 0,06 & $-0,16^{*}$ \\
\hline Morbilidade Psicológica & & $-0,04$ & 0,10 & $-0,03$ \\
\hline
\end{tabular}

Notas. IEI- Inibição da Exploração e Individualidade; QLE- Qualidade do Laço Emocional; ASD- Ansiedade de separação e Dependência.

$* p<0,05, * * * p<0,001$.

Tabela 4

Regressões Hierárquicas: Preditores do Estilo de Vida Incluindo as Variáveis de Apego ao Pai $(N=235)$

\begin{tabular}{|c|c|c|c|c|}
\hline Preditor & $\Delta R^{2}$ & $B$ & $E P B$ & $\beta$ \\
\hline Bloco 1 & 0,12 & & & \\
\hline Sexo & & $-4,86$ & 1,04 & $-0,30 * * *$ \\
\hline Idade & & $-0,55$ & 0,24 & $-0,15^{*}$ \\
\hline Tipo de família & & 0,24 & 1,54 & 0,01 \\
\hline Bloco 2 & 0,06 & & & \\
\hline Sexo & & $-5,41$ & 1,04 & $-0,33 * * *$ \\
\hline Idade & & $-0,56$ & 0,24 & $-0,15^{*}$ \\
\hline Tipo de família & & 0,53 & 1,57 & 0,02 \\
\hline IEI pai & & $-0,11$ & 0,05 & $-0,15^{*}$ \\
\hline QLE pai & & $-0,08$ & 0,07 & $-0,09$ \\
\hline ASD pai & & 0,04 & 0,07 & 0,05 \\
\hline Sintomatologia física & & $-0,12$ & 0,06 & $-0,15$ \\
\hline Morbilidade psicológica & & $-0,06$ & 0,10 & $-0,05$ \\
\hline
\end{tabular}

Notas. IEI- Inibição da Exploração e Individualidade; QLE- Qualidade do Laço Emocional; ASD- Ansiedade de separação e Dependência.

$* p<0,05, * * * p<0,001$. 


\section{Discussão}

Este estudo respondeu aos objetivos propostos, concluindo-se acerca da relação do apego aos pais, na idade adulta, com a sintomatologia física, a morbilidade psicológica e as características das relações amorosas. Constatou-se que o envolvimento numa relação romântica se reflecte na saúde, estando associado a um melhor estilo de vida e menos sintomas físicos. Além disso, permitiu conhecer as variáveis preditoras da sintomatologia física e do estilo de vida bem como a influência do sexo, tipo de família e NSE nas variáveis psicológicas em estudo. As análises revelaram que as mulheres apresentam mais sintomas físicos (Kroenke \& Spitzer, 1998; Wijk \& Kolk, 1997) e psicológicos do que os homens. Num grupo de jovens adultos, encontrou-se maior morbilidade psicológica (ansiedade e depressão) nas mulheres, sendo que esta relação tem vindo a ser encontrada consistentemente na literatura (Leach, Christensen, Mackinnon, Windsor, \& Butterworth, 2008). Claudino e Cordeiro (2006) avançam uma explicação para esta prevalência, arguindo que as mulheres serão mais sensíveis às exigências da sociedade, como resultado da maturação física e psíquica mais precoce, face aos homens.

No que respeita ao estilo de vida, os resultados deste estudo vão de encontro à literatura que encontra diferenças entre os sexos, sendo que as raparigas têm tendência a adotar menos comportamentos de risco (Colares, Franca, \& Gonzalez, 2009; Ribeiro \& Sousa, 2002). Deste modo, seria relevante aprofundar a relação entre sintomatologia física e estilo de vida, uma vez que as mulheres com comportamentos mais saudáveis apresentam também mais sintomas físicos (Galli, 2008). Este resultado pode dever-se às particularidades da amostra, já que os estudantes universitários experienciam elevados níveis de stress (Ferraz \& Pereira, 2002; Verger et al., 2009) que se podem manifestar em sintomas físicos (Cerchiari, Caetano, \& Faccenda, 2005) não invalidando, contudo, a adoção de um estilo de vida saudável, podendo constituir uma variável mediadora nesta relação.

A Ansiedade de Separação e Dependência (ASD) ao pai foi maior nas raparigas, contudo Assunção e Matos (2010) encontraram uma relação apenas entre a ASD dos rapazes em relação à mãe (i.e. a ansiedade de separação observava-se principalmente em relação ao progenitor do sexo oposto). No entanto, Moura e Matos (2008) encontraram maior ASD nas raparigas em relação a ambos os pais, o que segundo os autores pode indicar uma maior proximidade e dependência das raparigas na sua relação com os pais, face aos rapazes. Os jovens de famílias monoparentais percecionam maior inibição de exploração e individualidade (IEI) por parte da mãe do que os que pertencem a famílias intactas, o que parece sugerir que as mães que não vivem com o pai dos filhos (e.g. divórcio) são mais protetoras, restringindo mais a sua exploração em novos ambientes.
Verificou-se também que os jovens de famílias cujo NSE era médio/baixo apresentavam maior ASD em relação à mãe e maior conflito nas relações românticas, do que os de famílias de NSE elevado. A literatura que relaciona estas dimensões é, tanto quanto foi possível constatar, bastante limitada. Karney, Beckett, Collins, e Shaw (2007) sugerem que o nível socioeconómico tem influência nas relações amorosas na medida em que esta dimensão afeta o seu contexto imediato (e.g., relações familiares) e as suas características pessoais, como o estilo de apego, tendo o potencial de influenciar, na idade adulta, o modo como o indivíduo se envolve nas relações de compromisso. No que se refere à relação entre NSE e conflito, pode considerar-se que as crenças e comportamentos dos jovens acerca das relações amorosas são moldadas por diversos fatores, nomeadamente económicos, que definem as expectativas e comportamentos numa relação (Graber, Britto, \& Brooks-Gunn, 1999).

Vários autores têm associado os estilos de apego com a sintomatologia física (Wearden et al., 2005). Ciechanowski, Walker, Katon, e Russo (2002) concluíram que o apego inseguro se relacionava com maior sintomatologia física. Contudo, Myers e Vetere (2002) não encontraram esta relação. Neste estudo, mais sintomas físicos associaram-se a maior IEI por parte da mãe e do pai, mostrando que os jovens que vêem a sua individualidade mais restringida pelos pais têm mais sintomas físicos. Relativamente à morbilidade psicológica, os indivíduos com menor qualidade do laço emocional (QLE) em relação á mãe e pai e com maior IEI, por parte de ambos os pais, relatam mais sintomas psicológicos. Os resultados relativos à saúde física e psicológica podem ser explicados pelo facto de relações restritivas, superprotetoras e com baixo suporte, por parte dos pais, influenciarem a saúde, contribuindo para mais stress, depressão e sintomas físicos (Souza \& Baptista, 2008).

Os indivíduos menos satisfeitos com a sua relação romântica apresentam maior IEI por parte da mãe. Se considerarmos que estes participantes tendem a processar mais a informação negativa, isto pode aumentar a perceção da relação como mais insatisfatória e também contribuir para mais sintomas depressivos (Altin \& Terzi, 2010). A investigação tem demonstrado que os indivíduos envolvidos numa relação romântica apresentam melhor saúde física (Kiecolt-Glaser \& Newton, 2001) e menos comportamentos de risco (Braithwaite et al., 2010; Dennis, 2006), confirmando os resultados deste estudo.

Em relação aos preditores da sintomatologia fisica, a literatura dá conta da maior prevalência de sintomas físicos nas mulheres face aos homens, de forma relativamente conclusiva (Kroenke \& Spitzer, 1998; Wijk \& Kolk, 1997). Já ao nível do efeito da pertença a uma família monoparental, existem resultados contraditórios, mas no geral a investigação conclui que tem um efeito negativo na saúde física (Troxel \& Matthews, 2004). Os resultados deste estudo indicam que os indivíduos de 
Pereira, M. G., Ferreira, G. \& Paredes, A. C. (2013). Apego aos Pais, Relações Românticas, Estilo de Vida, Saúde Física e Mental em Universitários.

famílias intactas relatam menor sintomatologia física. No entanto, há estudos que não encontraram relação entre o tipo de família e este tipo de sintomas (Luecken \& Fabricius, 2003). Esta discrepância pode dever-se a variáveis moderadoras, como o conflito parental ou o distress causado pela separação, que influenciam a saúde física (Fabricius \& Luecken, 2007). Assim, não será apenas a configuração familiar a influenciar a saúde física, sendo necessário considerar outras variáveis, pelo que não é possível concluir se pertencer a uma família monoparental causa, em si, prejuízo a este nível. Menos divergente é a literatura que refere a existência de uma relação entre o estilo de vida e a saúde física (Berg et al., 2008; Leach et al., 2008). Numa revisão da literatura, constatou-se que os trabalhadores que desempenhavam funções profissionais sedentárias apresentavam maior prevalência de sintomas físicos, como dores lombares (Hildebrandt, Bongers, Dul, Dijk, \& Kemper, 2000). É possível integrar os jovens universitários neste cenário, já que a sua atividade consiste em assistir às aulas, trabalhar no computador, estudar ou seja, tarefas que são essencialmente sedentárias. Em relação à morbilidade psicológica, esta é muitas vezes apontada como preditora da sintomatologia física, uma vez que os indivíduos podem expressar as emoções negativas através da somatização (Kroenke, 2003).

No que diz respeito aos preditores de um estilo de vida saudável, as mulheres caracterizam-se pela adoção de comportamentos mais saudáveis do que os homens (Pereira \& Silva, 2002; Ribeiro \& Sousa, 2002). Um estudo realizado em estudantes universitários encontrou que o sexo é uma variável preditora de comportamentos de saúde, sendo as mulheres as que mais os adotam. Verificou-se que estudantes mais novos têm também mais comportamentos orientados para a saúde (Binkowska-Bury et al., 2010).

A literatura tem sugerido que o apego seguro é preditor de alimentação saudável (Faber, Dube, \& Belanger, 2009; Ribeiro \& Sousa, 2002). Sabendo que os comportamentos alimentares são parte do estilo de vida, um estudo verificou que quanto mais seguro o apego, mais saudável era a alimentação. Estes resultados sugerem que o apego influencia os comportamentos alimentares e, consequentemente, o estilo de vida (Faber et al., 2009). Faz assim sentido, neste estudo, a IEI pelos pais revelar-se preditora do estilo de vida.

\section{Considerações Finais}

O presente estudo evidenciou a importância das relações precoces na vida adulta ao nível da qualidade das relações românticas e da saúde física e mental. De acordo com os resultados, podem retirar-se implicações a nível desenvolvimental e clínico, uma vez que se concluiu acerca do impacto das relações precoces na idade adulta, salientando-se assim a necessidade de promover o estabelecimento de relações saudáveis entre os pais e as crianças, no sentido de contribuir para a qualidade da vida adulta. Torna-se também importante identificar as crianças que apresentam padrões de apego inseguros e dificuldades relacionais, no sentido de desenvolver programas de prevenção de dificuldades posteriores ao nível do apego nas relações românticas e estilo de vida bem como desenvolver programas de intervenção em jovens que incluam as questões do apego no sentido de promover a sua saúde física e psicológica.

Ao nível da investigação, seria importante a exploração das potenciais variáveis moderadoras e mediadoras nas relações encontradas, tais como se o apego funciona como uma variável mediadora na relação entre a saúde psicológica e a saúde física ou se tem um papel moderador na relação entre as características das relações amorosas e o estilo de vida.

As principais limitações a apontar a esta investigação são o facto de ter sido feita com uma amostra exclusivamente universitária e de apenas uma Universidade do Norte do Portugal. Relativamente aos instrumentos, algumas subescalas não foram utilizadas devido aos alfas de Cronbach serem inferiores aos valores aceitáveis. Além disso, o facto de os questionários, que avaliam o apego, serem de carácter retrospectivo pode também ser considerada uma limitação dado tratar-se dum método menos revelador que a entrevista (Bartholomew \& Horowitz, 1991).

\section{Referências}

Ainsworth, M. D., Blehar, M. C., Waters, E., \& Wall, S. (1978). Patterns of attachment. Hillsdale, NJ: Lawrence Erlbaum.

Altin, M., \& Terzi, Ş. (2010). How does attachment styles relate to intimate relationship to aggravate the depressive symptoms? Procedia Social and Behavioral Sciences, 2, 1008-1015.

Assunção, R., \& Matos, P. (2010). A vinculação parental e amorosa em adolescentes: O papel da competência interpessoal e da tomada de perspectiva. Actas do VII Simpósio Nacional de Investigação em Psicologia, 1574-1588.

Atkinson, L. (1997). Attachment and Psychopathology: From laboratory to clinic. In L. Atkinson \& K. J. Zucker (Eds.), Attachment and Psychopathology (pp. 3-16). New York: The Guilford Press.

Berg, T., Alavinia, S., Bredt, F., Lindeboom, D., Elders, L., \& Burdorf, A. (2008). The influence of psychosocial factors at work and life style on health, and work ability among professional workers. International Archives of Occupational and Environmental Health, 81, 1029-1036.

Berlin, L. J., Cassidy, J., \& Appleyard, K. (2008). The influence of early attachments on other relationships. In J. Cassidy \& P. R. Shaver (Eds.), Handbook of Attachment: Theory, Research and Clinical Applications (pp. 333-347). New York: The Guilford Press.

Binkowska-Bury, M., Kruk, W., Szymańska, J., Marć, M., PenarZadarko, B., \& Wdowiak, L. (2010). Psychosocial factors and health-related behavior among students from South-East Poland. Annals of Agricultural and Environmental Medicine, 17, 107-113.

Bowlby, J. (1984). Apego: Volume 1. Da trilogia apego e perda. São Paulo, SP: Martins Fontes.

Bowlby, J. (1985). Perda - Tristeza e depressão: Volume 3. Da 
trilogia apego e perda. São Paulo, SP: Martins Fontes.

Bowlby, J. (1998). Separação, angústia e raiva: Volume 2. Da trilogia apego e perda. São Paulo, SP: Martins Fontes.

Braithwaite, S. R., Delevi, R., \& Fincham, F. D. (2010). Romantic relationships and the physical and mental health of college students. Personal Relationships, 17, 1-12.

Cerchiari, E. A. N., Caetano, D., \& Faccenda, O. (2005). Prevalência de transtornos mentais menores em estudantes universitários. Estudos de Psicologia (Natal), 10(3), 413-420.

Ciechanowski, P., Walker, E., Katon, W., \& Russo, J. (2002). Attachment theory: A model for health care utilization and somatization. Psychosomatic Medicine, 64, 660-667.

Claudino, J., \& Cordeiro, R. (2006). Níveis de ansiedade e depressão nos alunos do curso de licenciatura em Enfermagem: O caso particular dos alunos da escola superior de saúde de Portalegre. Educação, Ciência e Tecnologia, 32, 197-210.

Colares, V., Franca, C., \& Gonzalez, E. (2009). Condutas de saúde entre universitários: Diferenças entre gêneros. Cadernos de Saúde Pública, 25(3), 521-528.

Collins, N. L., \& Read, S. J. (1990). Adult attachment, working models, and relationship quality in dating couples. Journal of Personality and Social Psychology, 58(4), 644-663.

Collins, W. A., \& Sroufe, L. A. (1999). Capacity for intimate relationships: A developmental construction. In W. Furman, B. B. Brown, \& C. Feiring (Eds.), The development of romantic relationships in adolescence (pp. 125-147). Cambridge, UK: Cambridge University Press.

Davis, K. E. (1996). The relationship rating form $(R R F)-A$ measure of the characteristics of romantic relationships and friendships. Retrieved November 13, 2010, from http://people. cas.sc.edu/daviske/LoveFriendsMeasure_rrf.pdf

De Haes, J. C., Olschewsky, M., Fayers, P., Visser, M. R., Cull, A., Hopwood, P., \& Sanderman, N. (1996). Measuring the quality of life of cancer patients with the Rotterdam symptom checklist (RSCL). Groningen, Netherlands: Northern Center for Healthcare Research, University of Groningen.

De Haes, J. C., van Knippenberg, F., \& Neijt, J. (1990). Measuring psychological and physical distress in cancer patients: Structure and application of the Rotterdam Symptom Checklist. British Journal of Cancer, 62, 1034-1038.

Dennis, M. R. (2006). Compliance and intimacy: Young adults' attempts to motivate health-promoting behaviors by romantic partners. Health Communication, 19(3), 259-267.

Faber, A., Dube, L., \& Belanger, A. (2009). Intergenerational study on the effects of attachment style on eating behaviors. Advances in Consumer Research, 36, 828-829.

Fabricius, W. V., \& Luecken, L. J. (2007). Postdivorce living arrangements, parent conflict, and long-term physical health correlates for children of divorce. Journal of Family Psychology, 21(2), 195-205.

Ferraz, M. F., \& Pereira, A. S. (2002). A dinâmica da personalidade e o homesickness (saudades de casa) dos jovens estudantes universitários. Psicologia, Saúde \& Doenças, 3(2), 149-164.

Field, A. (2009). Discovering statistics using SPSS (and sex and drugs and rock ' $n$ ' roll). London: Sage.

Galli, K. S. (2008). Diferenças de gênero relacionadas ao estilo de vida, estado de saúde e utilização dos serviços de saúde: Um inquérito de base populacional (Dissertação de mestrado, Universidade do Vale do Itajaí, Itajaí, SC, Brasil).

Gouveia, J. (2004). Diferenças ao nível do género na adaptação psicossocial a curto prazo no pós enfarte agudo do miocárdio (Dissertação de mestrado, Universidade do Minho, Braga,
Portugal)

Graber, J. A., Britto, P. R., \& Brooks-Gunn, J. (1999). What's love got to do with it? Adolescents' and young adults' beliefs about sexual and romantic relationships. In W. Furman, C. Feiring, \& B. B. Brown (Eds.), The development of romantic relationships in adolescence (pp. 364-395). New York: Cambridge University.

Green, L., \& Anderson, C. (1986). Community health. St. Louis, MO: Times Mirror.

Hale, C. J. (2006). Daily stress and physical health: The role of intimacy and conflict in romantic relationships. Dissertation Abstracts International, 66(12-A), 42-97.

Hazan, C., \& Shaver, P. (1987). Romantic love conceptualized as an attachment process. Journal of Personality and Social Psychology, 52(3), 511-524.

Hildebrandt, V. H., Bongers, P. M., Dul, J., Dijk, F. J., \& Kemper, H. C. (2000). The relationship between leisure time, physical activities and musculoskeletal symptoms and disability in worker populations. International Archives of Occupational and Environmental Health, 73, 507-518.

Holmes, J. (1993). John Bowlby \& Attachment theory. London: Routledge.

Karney, B., Beckett, M., Collins, R., \& Shaw, R. (2007). Adolescent romantic relationships as precursors of healthy adult marriages - A review of theory, research, and programs. Santa Monica, CA: Rand.

Kiecolt-Glaser, J. K., \& Newton, T. L. (2001). Marriage and health: His and hers. Psychological Bulletin, 127, 472-503.

Kroenke, K. (2003). The interface between physical and psychological symptoms. Primary Care Companion Journal of Clinical Psychiatry, 5(7), 11-18.

Kroenke, K., \& Spitzer, R. (1998). Gender differences in the reporting of physical and somatoform symptoms. Psychosomatic Medicine, 60, 150-155.

Leach, L., Christensen, H., Mackinnon, A., Windsor, T., \& Butterworth, P. (2008). Gender differences in depression and anxiety across the adult lifespan: The role of psychosocial mediators. Social Psychiatry and Psychiatric Epidemiology, 43, 983-998.

Lind, W. R. (2008). Casais biculturais e monoculturais: Diferenças e recursos (Tese de doutorado, Universidade de Lisboa, Portugal).

Luecken, L., \& Fabricius, W. (2003). Physical health vulnerability in adult children from divorced and intact families. Journal of Psychosomatic Research, 55, 221-228.

Markey, C., Markey, P., \& Gray, H. (2007). Romantic relationships and health: An examination of individuals' perceptions of their romantic partners' influences on their health. Sex Roles, 57, 435-445.

Maroco, J. (2007). Análise estatística com utilização do SPSS. Lisboa, Portugal: Silabo.

Matos, P. (2002). (Des) continuidades na vinculção aos pais e ao par amoroso em adolescentes (Tese de doutorado, Universidade do Porto, Portugal).

Matos, P. M., \& Costa, M. E. (2001). Questionário de vinculação ao pai e à mãe: Versão III. Manuscrito não publicado.

McIntyre, M. T., Pereira, G., Soares, V., Gouveia, J., \& Silva, S. (1999). Escala de Ansiedade e Depressão Hospitalar. Versão Portuguesa de investigação. Braga, Portugal: Departamento de Psicologia, Universidade do Minho.

Monteiro, S., Tavares, J., \& Pereira, A. (2007). Relação entre vinculação, sintomatologia psicopatológica e bem-estar em estudantes do primeiro ano do ensino superior. Psicologia, 
Pereira, M. G., Ferreira, G. \& Paredes, A. C. (2013). Apego aos Pais, Relações Românticas, Estilo de Vida, Saúde Física e Mental em Universitários.

Saúde \& Doenças, 8(1), 83-93.

Moreira, J. M., Lind, W., Santos, M. J., Moreira, A. R., Gomes, M. J., Justo, J., ...Faustino, M. (2006). "Experiências de relações próximas", um questionário de avaliação das dimensões básicas dos estilos de apego nos adultos: Tradução e validação para a população portuguesa. Laboratório de Psicologia, 4(1), 3-27.

Morgan, J. M., \& Shaver, P. R. (1999). Attachment processes and commitment to romantic relationships. In J. M. Adams \& H. Jones (Eds.), Handbook of Interpersonal Commitment and Relationship Stability (pp. 109-124). New York: Kluwer Academic.

Moura, O., \& Matos, P. (2008). Apego aos pais, divórcio e conflito interparental em adolescentes. Psicologia, 22(1), 127-152.

Myers, L. B., \& Vetere, A. (2002). Adult romantic attachment styles and health-related measures. Psychology, Health \& Medicine, 7(2), 175-180.

Pedras, C. S. (2009). Variáveis de saúde, familiares e de psicopatologia em filhos de veteranos da guerra colonial portuguesa (Dissertação de mestrado, Universidade do Minho, Braga, Portugal)

Pereira, M. G., Ferreira, G., \& Paredes, A. C. (2010). Questionário sociodemográfico. Versão de Investigação. Braga, Portugal: Escola de Psicologia, Universidade do Minho.

Pereira, M. G., \& Pedras, S. (2008). Questionário de Estilo de Vida. Manuscrito não publicado, Universidade do Minho, Braga, Portugal.

Pereira, M. G., \& Silva, S. (2002). Atribuição de sintomas, comportamentos de saúde e adesão em utentes de centro de saúde da zona norte. Análise Psicológica, 1(20), 35-43.

Ribeiro, J. P., \& Sousa, M. (2002). Apego e comportamentos de saúde: Estudo exploratório de uma escala de avaliação do apego. Análise Psicológica, 1, 67-75.

Rocha, M. (2008). O desenvolvimento das relações de apego na adolescência: Associações entre contextos relacionais com pais, pares e par amoroso (Tese de doutorado, Universidade do Porto, Portugal).

Santos, M. J., Costa, F. L., Watson, M., Geer, S., Haes, J., Knippenberg, F. V., ...Borne, B. V. (1994). Adaptação psicológica e qualidade de vida em doentes oncológicos: Escalas de avaliação. Revista de Psiquiatria Clínica, 15(1), 25-36.

Simon, R., \& Barrett, A. (2010). Nonmarital romantic relationships and mental health in early adulthood: Does the association differ for women and men? Journal of Health and Social Behavior, 51(2), 168-182.

Simpson, J. A. (1990). Influence of attachment styles on romantic relationships. Journal of Personality and Social Psychology, 59(5), 971-980.

Souza, M. S., \& Baptista, M. N. (2008). Associações entre suporte familiar e saúde mental. Psicologia Argumento, 26(54), 207-215.

Troxel, W. M., \& Matthews, K. A. (2004). What are the costs of marital conflict and dissolution to children's physical health? Clinical Child and Family Psychology Review, 7(1), 29-57.

Verger, P., Combes, J., Kovess-Masfety, V., Choquet, M., Guagliardo, V., Rouillon, F., \& Peretti-Wattel, P. (2009). Psychological distress in first year university students: Socioeconomic and academic stressors, mastery and social support in young men and women. Social Psychiatry and Psychiatric Epidemiology, 44, 643-650.

Wearden, A. J., Lamberton, N., Crook, N., \& Walsh, V. (2005). Adult attachment, alexithymia, and symptom reporting - An extension to the four category model of attachment. Journal of Psychosomatic Research, 58, 279-288.

Wijk, C., \& Kolk, A. (1997). Sex differences in physical symptoms: The contribution of symptom perception theory. Social Science and Medicine, 45(2), 231-246.

Zigmond, A. S., \& Snaith, R. P. (1983). The hospital anxiety and depression scale. Acta Psychiatrica Scandinavica, 67, 361-370.
Recebido: 08/09/2011

$1^{a}$ revisão: 02/07/2012

$2^{a}$ revisão: 25/07/2012

Aceite final: $21 / 08 / 2012$ 